\title{
Behaviour of Induction Machines under Fault Conditions - Application of the Instantaneous Symmetrical Components Method
}

\author{
Essam S Hamdi \\ School of Science, Engineering and Design \\ Teesside University, Middlesbrough, UK \\ e.hamdi@tees.ac.uk
}

\begin{abstract}
Although the method of symmetrical components is commonly used in investigating unbalanced operation of electrical machines and power systems, its application in the analysis of transient behaviour of electrical machines can be advantageous. The main advantage lies in the ease with which different fault conditions can be analysed. In this paper, the differential equations describing an induction machine are developed in terms of the symmetrical components of the instantaneous values of transient voltages and currents. The developed model enables investigation of various fault conditions when the machine is operating as a motor or a generator.
\end{abstract}

Index Terms-Symmetrical components, induction machines, transient analysis, fault conditions, asymmetrical faults, dynamic braking.

\section{NOMENCLATURE}

$v, i \quad$ Instantaneous voltage and current

$\alpha, \beta \quad$ Subscripts indicating stator and rotor

$L_{\alpha}, L_{\beta} \quad$ Stator and rotor windings self inductance

$L_{m} \quad$ Magnetising or mutual inductance

$\vartheta \quad$ Rotor angular position with respect to stator

$v \quad$ Ratio of rotor speed to synchronous speed

$\psi \quad=e^{j(2 \pi / 3)}$

$\tau \quad=\omega t$

$\omega \quad$ Supply frequency, $\mathrm{rad} / \mathrm{s}$

s $\quad$ Per-unit slip $=1-v$

$D \quad$ Differential operator with respect to $(\omega t)$

$0,1,2$ Subscripts indicating zero, positive and negative sequence

\section{INTRODUCTION}

Developed by Fortescue [1] a century ago, the method of symmetrical components (or coordinates) has been extensively applied in the analysis of electrical systems. The application of this method of analysis has led to a greater understanding of both power system component and network behaviour, giving rise to significant developments in the associated technology.

In the conventional application of the symmetrical components method (SCM), the phasors representing the three-phase voltages of an unbalanced electrical system are resolved into their symmetrical components; each acting on the corresponding sequence network [2]. Over the years, the method has been extended to cater for distribution networks combining one-phase and two-phases [3]-[5].

Recently, SCM has been modified and utilised in the analysis of electrical machines of special designs and/or under fault conditions. For example, the analysis of unbalanced operation of 5-phase induction machines is presented in [6] and the performance of a class of 5-phase permanent-magnet synchronous-reluctance motors is evaluated, with one open phase, in [7]. The method has also facilitated development of fault signature analyses of both synchronous and induction machines [8]-[10]. The application of the SCM to the analysis of electrical machines has, in most cases, been performed in terms of complex quantities representing relevant phasors [6][10].

Although the concept of instantaneous symmetrical components appear in literature, it is used to indicate that the transformation is performed to variables, on phasor forms obtained at discrete instants during the computation cycle [11][13]. The approach adopted here is quite different: it relies on the derivation of the induction machine's differential equations in terms of the instantaneous values of (not the phasors representing) phase voltages and currents. Different operating conditions, including symmetrical and asymmetrical faults, and dynamic braking, can then be investigated by modifying the machine's differential equations accordingly. The analysis is developed in terms of the ratio of rotor-speed to synchronous-speed, and this enables investigation of both the motoring and generating modes.

\section{TRANSIENT ANALYSIS}

When the operating condition of an electrical machine abruptly changes, its currents and voltages assume new values in order to satisfy the laws of physics that govern the new condition. In an electrical machine, the energy stored in the magnetic field can't change suddenly and, therefore, transient current and voltage components are produced and, while they will rapidly diminish, they may reach levels so high that the windings become degraded or even damaged. Therefore, estimating the transient conditions and identification of design parameters that affect their value and duration is an important engineering topic. 
The symmetrical components of the instantaneous values of transient voltages and current are obtained following the same process used when the method is applied to phasors. For example, if $v_{a}, v_{b}$ and $v_{c}$ are the instantaneous values of voltages of a 3-phase system, the sequence components of $v_{a}$ are readily expressed as:

$v_{a 0}=\frac{1}{3}\left(v_{a}+v_{b}+v_{c}\right)$

$v_{a 1}=\frac{1}{3}\left(v_{a}+\psi v_{b}+\psi^{2} v_{c}\right)$

$v_{a 2}=\frac{1}{3}\left(v_{a}+\psi^{2} v_{b}+\psi v_{c}\right)$

Unlike the phasor voltages, the instantaneous values $v_{a}, v_{b}$ and $v_{c}$ are real quantities. Since $\psi$ and $\psi^{2}$ are conjugate vectors, the positive-sequence component $v_{a 1}$ and the negative-component $v_{a 2}$ are also, always, conjugate vectors. Therefore, if one component is determined, the other is readily determined. Indeed, this is one of the advantages of adopting the treatment presented here.

Another advantage lies in the ease with which different fault conditions can be analysed. For example, to investigate a shortcircuit between two phases, say phase $b$ and $c$, we express $v_{b c}$ in terms of the sequence voltages and determine the condition(s) under which it vanishes. In terms of the sequence components, the phase voltages are:

$v_{a}=v_{a 0}+v_{a 1}+v_{a 2}$

$v_{b}=v_{a 0}+\psi^{2} v_{a 1}+\psi v_{a 2}$

$v_{c}=v_{a 0}+\psi v_{a 1}+\psi^{2} v_{a 2}$

The line voltage $v_{b c}$ is:

$v_{b c}=\left(\psi^{2}-\psi\right) v_{a 1}+\left(\psi-\psi^{2}\right) v_{a 2}=-j \sqrt{3}\left(v_{a 1}-v_{a 2}\right)$

Consequently, if the terminal $b$ and $c$ are shorted:

$v_{a 1}=v_{a 2}$

Relevant response can be obtained when this condition is introduced to the machine's differential equations. Similarly, different fault conditions can easily be investigated.

\section{MODEL DEVELOPMENT}

With reference to Fig.1, the terminal voltage of a stator (or rotor) phase would be equal to the resistance drop plus the voltage induced by the flux-linkage variation with time. Therefore, for the $a$-phase stator winding, the positivesequence voltage is:

$v_{\alpha 1}=\left(r_{\alpha}+L_{\alpha} \frac{d}{d t}\right) i_{\alpha 1}+M_{\beta} \frac{d}{d t}\left(i_{\beta 1} e^{j \vartheta}\right)$

The corresponding negative-sequence equation is, of course, the conjugate of the positive-sequence equation, or:

$v_{\alpha 2}=\left(r_{\alpha}+L_{\alpha} \frac{d}{d t}\right) i_{\alpha 2}+M_{\beta} \frac{d}{d t}\left(i_{\beta 2} e^{-j \vartheta}\right)$

Similarly, the corresponding equations for the rotor winding are:

$v_{\beta 1}=\left(r_{\beta}+L_{\beta} \frac{d}{d t}\right) i_{\beta 1}+M_{\alpha} \frac{d}{d t}\left(i_{\alpha 1} e^{-j \vartheta}\right)$ and,

$v_{\beta 2}=\left(r_{\beta}+L_{\beta} \frac{d}{d t}\right) i_{\beta 2}+M_{\alpha} \frac{d}{d t}\left(i_{\alpha 2} e^{j \vartheta}\right)$

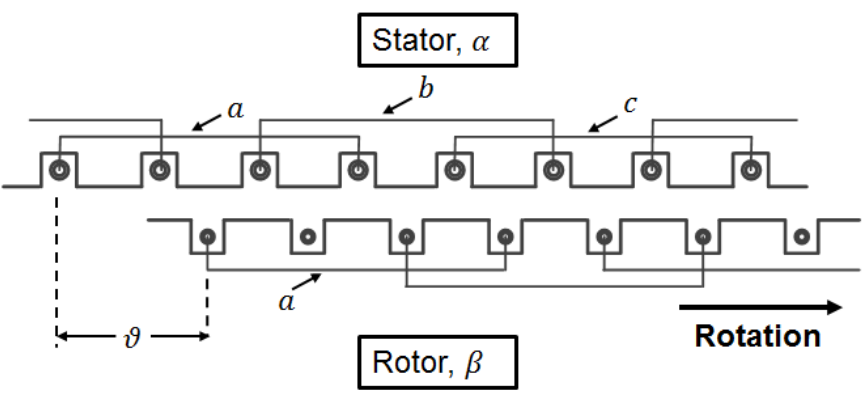

Fig. 1. A developed diagram of a 3-phase induction machine.

Noting that, in the absence of saliency, $M_{\alpha}=M_{\beta}=M$, and introducing the differential operator $D$, we obtain:

$$
\begin{aligned}
& v_{\alpha 1}=\left(r_{\alpha}+x_{\alpha} D\right) i_{\alpha 1}+x_{m} D\left(i_{\beta 1} e^{j \vartheta}\right) \\
& v_{\beta 1}=\left(r_{\beta}+x_{\beta} D\right) i_{\beta 1}+x_{m} D\left(i_{\alpha 1} e^{-j \vartheta}\right)
\end{aligned}
$$

If the speed is assumed constant during the transient period: $\vartheta=v \omega t=v \tau$ where $v=n / n_{s}$, then:

$D\left(i_{\alpha 1} e^{-j v \tau}\right)=e^{-j v \tau} D i_{\alpha 1}-j v e^{-j v \tau} i_{\alpha 1}=e^{-j v \tau}(D-j v) i_{\alpha 1}$ $D\left(i_{\beta 1} e^{j v \tau}\right)=e^{j v \tau} D i_{\beta 1}+j v e^{j v \tau} i_{\beta 1}$ or,

$D i_{\beta 1}=e^{-j v \tau}(D-j v)\left(i_{\beta 1} e^{j v \tau}\right)$

Eq. (2) can now be expressed as:

$v_{\beta 1}=e^{-j v \tau}\left[r_{\beta}+x_{\beta}(D-j v)\right]\left(i_{\beta 1} e^{j v \tau}\right)+e^{-j v \tau} x_{m}(D-j v) i_{\alpha 1}$

or,

$$
v_{\beta 1} e^{j v \tau}=\left[r_{\beta}+x_{\beta}(D-j v)\right]\left(i_{\beta 1} e^{j v \tau}\right)+x_{m}(D-j v) i_{\alpha 1}
$$

Again, the corresponding negative-sequence equation is the conjugate of the positive-sequence equation:

$v_{\beta 2} e^{-j v \tau}=\left[r_{\beta}+x_{\beta}(D+j v)\right]\left(i_{\beta 1} e^{-j v \tau}\right)+x_{m}(D+j v) i_{\alpha 1}$

During normal motor operation, the rotor circuit is shorted (i.e. $v_{\beta 1}=0$ ). Solving (1) and (3) for $i_{\alpha 1}$ we get:

$$
\begin{array}{r}
\left\{\left(r_{\alpha}+x_{\alpha} D\right)\left[r_{\beta}+x_{\beta}(D-j v)\right]-x_{m}^{2} D(D-j v)\right\} i_{\alpha 1} \\
=\left[r_{\beta}+x_{\beta}(D-j v)\right] v_{\alpha 1}
\end{array}
$$

Dividing by $x_{\alpha} x_{\beta}$, the differential equation for the positivesequence stator current becomes:

$$
\begin{aligned}
{\left[D^{2}+\left(\frac{k_{\alpha}}{\sigma}+\frac{k_{\beta}}{\sigma}-j v\right) D+\frac{k_{\alpha}}{\sigma}\right.} & \left.\left(k_{\beta}-j v\right)\right] i_{\alpha 1} \\
& =\left[k_{\beta}+D-j v\right] \frac{v_{\alpha 1}}{\sigma x_{\alpha}}
\end{aligned}
$$

where: $k_{\alpha}=\frac{r_{\alpha}}{x_{\alpha}}$ and $k_{\beta}=\frac{r_{\beta}}{x_{\beta}}$ and $\sigma=1-\frac{x_{m}^{2}}{x_{\alpha} x_{\beta}}$

Sine Eq. (5) is linear with constant coefficients, the general expression for the transient currents is:

$i_{\alpha 1}=C_{\alpha 1} e^{p_{1} \tau}+C_{\alpha 2} e^{p_{2} \tau}$ 
where $p_{1}$ and $p_{2}$ are the roots of the characteristic equation:

$$
p^{2}+\left(\frac{k_{\alpha}}{\sigma}+\frac{k_{\beta}}{\sigma}-j v\right) p+\frac{k_{\alpha}}{\sigma}\left(k_{\beta}-j v\right)=0
$$

It should be noted that subscripts attached to the complementary function coefficient $C_{\alpha}$ don't signify positive or negative sequence but corresponds to the subscript used to designate the different roots of the characteristic equation.

The characteristic equation for the negative-sequence current is readily obtained as the conjugate of Eq. (6). The roots of Eq. (6) are:

$p=-\frac{k_{\alpha}+k_{\beta}}{2 \sigma}+j \frac{v}{2} \mp \sqrt{\left(\frac{k_{\alpha}+k_{\beta}}{2 \sigma}-j \frac{v}{2}\right)^{2}-\frac{k_{\alpha}}{\sigma}\left(k_{\beta}-j v\right)}$

In general, the roots of the characteristic equation are complex numbers and, therefore, the response is a damped sinusoid.

\section{RESUlts}

By a way of an example, the case of a simultaneous 3-phase short circuit at the stator terminal is investigated. This fault condition can be simulated by applying a positive-sequence terminal voltage $-v_{\alpha 1}$ in series with the voltage $v_{\alpha 1}$ (the voltage acting on the machine prior to the short circuit). Therefore, the current immediately after the fault consists of two components: the steady-state current due to $v_{\alpha 1}$ and the current caused by sudden application of $-v_{\alpha 1}$. Immediately after short-circuit, the only transient currents are those caused by the application of $-v_{\alpha 1}$ at the stator terminal. Substituting in Eq. (5), we obtain:

$$
\begin{aligned}
{\left[D^{2}+\left(\frac{k_{\alpha}}{\sigma}+\frac{k_{\beta}}{\sigma}-j v\right) D\right.} & \left.+\frac{k_{\alpha}}{\sigma}\left(k_{\beta}-j v\right)\right] i_{\alpha 1} \\
& =\left[k_{\beta}+D-j v\right] \frac{\left(-v_{\alpha 1}\right)}{\sigma x_{\alpha}}
\end{aligned}
$$

It should be noted that the corresponding characteristic equation still has the same roots as expressed in Eq. (7).

Assuming that $v_{\alpha 1}=V_{\alpha 1} e^{j \tau}$, the positive-sequence component of the stator current can be expressed as:

$$
i_{\alpha 1}=\left[\frac{\left(k_{\beta}+p_{1}-j v\right)}{p_{1}-p_{2}} \frac{e^{p_{1} \tau}}{p_{1}-j}+\frac{\left(k_{\beta}+p_{2}-j v\right)}{p_{2}-p_{1}} \frac{e^{p_{2} \tau}}{p_{2}-j}\right] \frac{\left(-V_{\alpha 1}\right)}{\sigma x_{\alpha}}
$$

where $p_{1}$ and $p_{2}$ are the roots of the characteristic equation (6).

\section{A. Machine Directly Connected to Supply}

If the short occurs at the instant when the phase voltage is maximum, $\left(V_{\alpha}\right)$, then $V_{\alpha 1}=0.5 V_{\alpha}$. Alternatively, if the fault occurs when the phase voltage is zero and rising, then $V_{\alpha 1}=$ $-j 0.5 V_{\alpha}$

In normal operation, the rotor speed changes only a few percent from full-load motor action to full-load generator action. Therefore, the effect of rotor speed on the transient current response is, firstly, investigated. The data of the test machine are summarised in Table 1.
Using the data of Table 1, Figs. 2 and 3 are obtained for the cases when the stator winding is shorted, at the instant of maximum and minimum phase voltage; respectively. It shows the variation of the fault current with time (expressed as $\omega t$ and converted to degrees).

In an induction machine, transient currents and voltages are due to the energy stored in the magnetic field and can be approximated to $\frac{1}{2} L i^{2}$. As the phase current lags the voltage, the current, and the stored magnetic energy, at the voltage zero cross-over point is expected to be higher than when the phase voltage is at a maximum. This explains why the peak fault current of Fig. 3 is about twice that of Fig. 2.

TABLE I

DATA OF TEST MACHINE AT RATED FREQUENCY

\begin{tabular}{|l|l|}
\hline Stator resistance, $r_{\alpha}$ & $0.025 \mathrm{pu}$ \\
\hline Stator winding reactance, $x_{\alpha}$ & $2.8 \mathrm{pu}$ \\
\hline Rotor resistance, $r_{\beta}$ & $0.025 \mathrm{pu}$ \\
\hline Stator winding reactance, $x_{\beta}$ & $2.8 \mathrm{pu}$ \\
\hline Magnetising reactance, $x_{m}$ & $2.66 \mathrm{pu}$ \\
\hline Leakage coefficient, $\sigma$ & $0.1 \mathrm{pu}$ \\
\hline
\end{tabular}

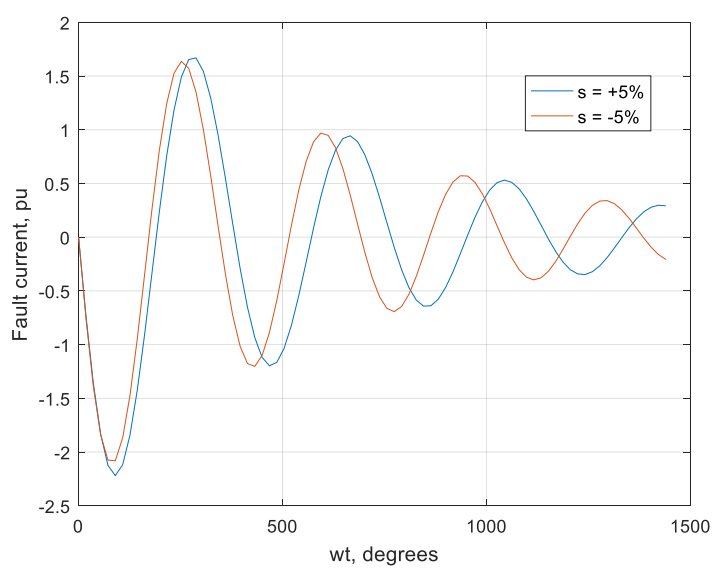

Fig. 2. Variation of short-circuit current with slip - fault at the instant of maximum phase voltage.

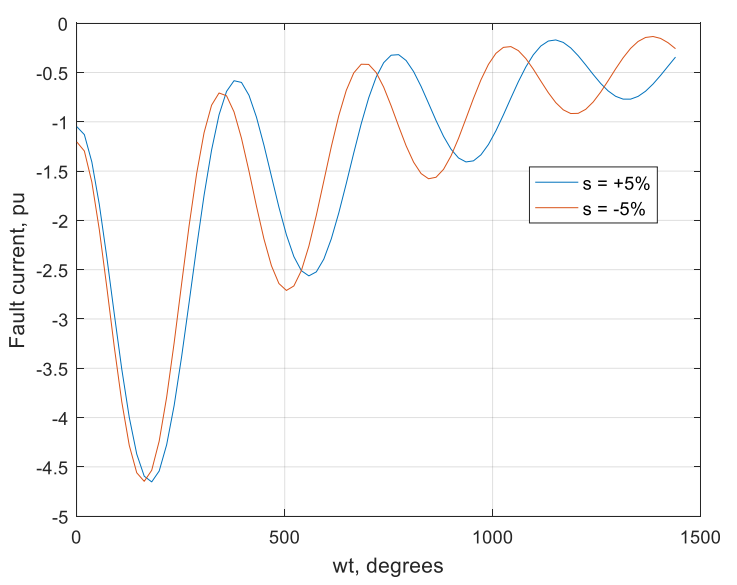

Fig. 3. Variation of short-circuit current with slip - fault at the instant of zero (rising) phase voltage. 


\section{B. Effect of Supply Impedance}

The effect of adding an impedance to the stator winding is considered. This case is of practical significance as the machine may be connected to source through a transformer or other auxiliary circuits.

Fig. 4 shows the effect of increasing the stator resistance on the transient response. It is seen that the effect is to increase the damping coefficient. More significantly, the peak fault current is only marginally affected by the stator resistance.

A more practical condition is to consider adding a supply transformer inductance. The corresponding response is shown in Fig. 5, where the transformer reactance is taken at $0.15 \mathrm{pu}$.

It is seen that the effect of the supply inductance is two fold. Firstly, the peak transient current (which still occurs at about quarter of a cycle) is reduced by about $30 \%$. Secondly, as the resistance considered is only that of the machine, the damping coefficient is significantly reduced and the oscillatory current persists for about 6 cycles.

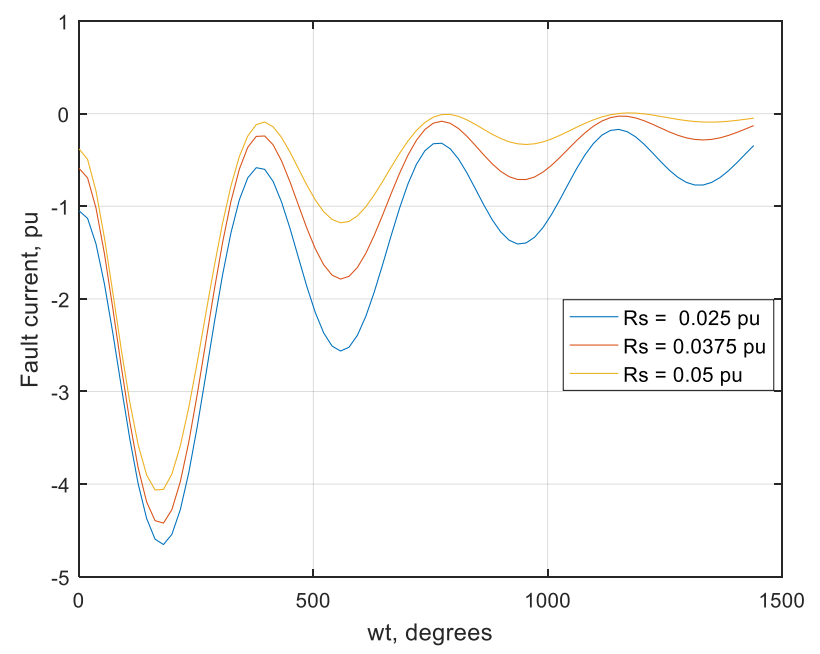

Fig. 4. Effect of stator resistance on fault current.

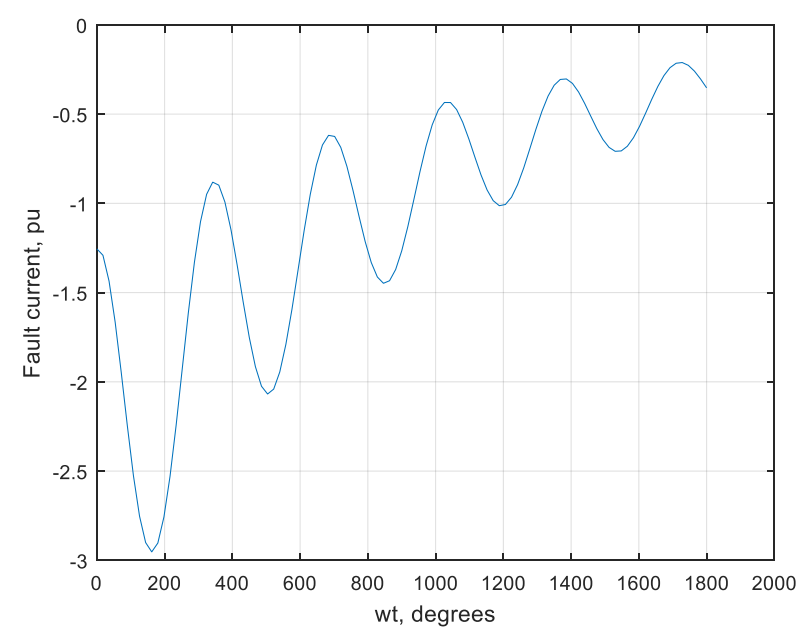

Fig. 5. Effect of supply transformer inductance on fault current.

\section{CONCLUSIONS}

In this paper, the induction machine's differential equations are derived in terms of the instantaneous values of phase voltages and currents. Such an approach enables investigation of different operating conditions, including symmetrical and asymmetrical faults, and dynamic braking, by modifying the machine's differential equations accordingly. The machine's equations are developed in terms of the ratio of rotor-speed to synchronous-speed, and this facilitates investigation of both the motoring and generating modes.

Although results are presented for the case of a simultaneous 3-phase short circuit at the stator terminals, the machine's equations can be readily modified to represent asymmetrical faults. The effect of stator winding resistance on fault levels is shown to be minimal. On the other hand, the effect of supply transformer reactance is shown to affect both the fault current level and duration.

\section{REFERENCES}

[1] C. L. Fortescue, "Method of Symmetrical Co-Ordinates Applied to the Solution of Polyphase Networks," Trans. AIEE, vol. XXXVII, no. 2, pp. 1027-1140, July 1918.

[2] M. A. Laughton, "Analysis of unbalanced polyphase networks by the method of phase co-ordinates - part 1: System representation in phase frame of reference," Proc. IEE, vol. 115, no. 8, pp. 1163-1172, Aug.1968.

[3] I. Dzafic et al, "Three-Phase Power Flow in Distribution Networks using Fortescue Transformation,” IEEE Trans. Power Syst., vol. 28, no. 2, pp. 1027-1034, May 2013

[4] R. A. Jabr, and I. Dzafic, "A Fortescue Approach for Real-Time Short Circuit Computation in Multiphase Distribution Networks," IEEE Trans. Power Syst., vol. 30, no. 6, pp. 3276-3285, Nov. 2015.

[5] Y. Gu, W. Li and X. He, "Analysis and Control of Bipolar LVDC Grid with DC Symmetrical Component Method", IEEE Trans. Power Syst., vol. 31, no. 1, January 2016.

[6] L. A. Pereira, L. F. A. Pereira, S. Haffner and G. Nicol, "Unbalanced operation of five-phase induction machines using steady state symmetrical components-part I: theoretical considerations", 41st Annual Conf. of the IEEE Industrial Electronics Society (IECON2015), 2015 .

[7] A. K. M. Arafat, S. Choi and J. Baek, "Open Phase Fault Detection of a Five-Phase Permanent Magnet Assisted Synchronous Reluctance Motor based on Symmetrical Components Theory", IEEE Trans. on Industrial Electronics, Volume: PP, Issue: 99 (Early Access Article), 2017.

[8] C. P. Salomon et al, "Discrimination of Synchronous Machines Rotor Faults in Electrical Signature Analysis based on Symmetrical Components", IEEE Transactions on Industry Applications, Volume PP, Issue 99 (Early Access Article), 2016.

[9] V. R. Cociu, L. Cociu and B. Vladimir, "Symmetrical Components Expression of the Stator Current of an Induction Machine under Internal Fault", Proc. of 2016 International Conf. and Exposition on Electrical and Power Engineering (EPE 2016), 20-22 October, Iasi, Romania.

[10] C.T. Kowalski, M. Wolkiewicz, "Stator Faults Diagnosis of Converterfed Induction Motor using Symmetrical Components and Neural Networks", Proc. of European Power Electronics Application Conf. EPE'2009.

[11] M. I. Marei, E. F. El-Saadany, and M. M. A. Salama, "A new approach to control DVR based on symmetrical components estimation," IEEE Trans. Power Del., vol. 22, no. 4, pp. 2017-2024, Oct. 2007.

[12] G. Chen et al, "A Novel SPLL and Voltage Sag Detection Based on LES Filters and Improved Instantaneous Symmetrical Components Method", IEEE Trans. on Power Electronics, vol. 30, no. 3, March 2015.

[13] N. R. Tummuru, M. K. Mishra and S. Srinivas, "Multifunctional VSC Controlled Microgrid Using Instantaneous Symmetrical Components Theory", IEEE Trans. on Sustainable Energy, vol. 5, no. 1, January 2014. 